\title{
Solute Adsorption and Gel Layer Formation during Ultrafiltration of Ovalbumin
}

\author{
Hiroshi Nabetani $^{1 *}$, Mitsutoshi Nakajima ${ }^{1}$, Shoji Hagiwara ${ }^{1}$, Atsuo Watanabe $^{2}$, Shin-ichi NaKao ${ }^{3}$ and \\ Shoji KIMURA $^{3}$ \\ ${ }^{1}$ Food Engineering Division, National Food Research Institute, NARO, 2-1-12, Kan-nondai, Tsukuba, Ibaraki 305-8642 Japan
${ }^{2}$ Membrane \& Separation Research Circle of Food, 1-1-3-3321, Kawaguchi, Kawaguchi 332-0015 Japan
${ }^{3}$ Department of Chemical System Engineering, The University of Tokyo, 7-3-1 Hongo, Bunkyo-ku, Tokyo $163-8677$ Japan
}

Received October 1, 2008; Accepted February 5, 2009

To define the influence of osmotic pressure, solute adsorption, and gel layer formation on permeate flux during the ultrafiltration of an ovalbumin solution, the characteristics of the gel-like layer formed on the membrane surface were investigated and the adsorption rate of ovalbumin to membranes was measured under different conditions. Gel-like layer observed on the membrane surface was found to be a deposit layer formed by an aggregate of denatured ovalbumin which could be precipitated by centrifugation. This layer had a rejection ability for ovalbumin itself and little resistance for permeate flow. In both cases where there is a layer and where is not, the flux values during the ultrafiltration could be precisely predicted by the osmotic pressure and adsorption resistance model which took into account the influence of osmotic pressure and solute adsorption. The forced flow of solution into membrane pores shortened the time to reach adsorption equilibrium, suggesting a notable influence of solute adsorption into membrane pores.

Keywords: ovalbumin, osmotic pressure, adsorption, gel layer, ultrafiltration

\section{Introduction}

It is important to estimate the permeate flux decline caused by fouling phenomena in ultrafiltration processes. In our previous work (Nabetani et al., 1990), the permeate flux values during ultrafiltration of an ovalbumin solution were analyzed and found to be precisely predicted by an osmotic pressure and adsorption resistance model. This model takes into account the influence of an increase in osmotic pressure at the membrane surface caused by concentration polarization phenomena and the extra resistance caused by solute adsorption, but disregards any effect of the gel-like layer, which was observed on the membrane surface. Previously, the gel layer had been regarded to be the main factor limiting flux values (Nakao et al., 1979; Matsumoto et al., 1987).

Since the osmotic pressure and adsorption resistance model can accurately predict permeate flux, some questions arise:

What is the gel-like layer? Although there is no influence of the gel-like layer on flux in the model, this layer was

*To whom correspondence should be addressed.

E-mail: nabetani@affrc.go.jp always observed on the membrane surface after ultrafiltration of an ovalbumin solution.

When a gel-like layer was observed on membrane surfaces, the gel polarization model (Baker and Strathaman, 1970; Blatt et al., 1970; Porter, 1972) had usually been employed for analysis of permeate flux during ultrafiltration of macromolecules. In analysis with the gel polarization model, permeate flux $J_{v}$ is expressed as

$$
J_{\mathrm{v}}=k \ln \left(C_{\mathrm{g}} / C_{\mathrm{b}}\right)
$$

where $k$ and $C_{\mathrm{b}}$ are the mass transfer coefficient in concentration polarization layer and concentration of the feed solution, respectively; where concentration of the gel layer formed on the membrane surface, $C_{\mathrm{g}}$, is assumed to be constant and dependent only on solute type and not on filtration conditions. However, Nakao et al. (1979) pointed out that the assumption bore several contradictions, and tried to measure the concentration of the gel layer by scraping out the layer from a membrane surface just after ultrafiltration of ovalbumin or polyvinylalcohol. The experimental results revealed that the concentration of the gel layer was not constant and depended on experimental conditions such as feed concentration and 
feed flow rate. Subsequently, they proposed the following model which provided the permeate flux value as,

$$
J_{\mathrm{v}}=\Delta P /\left(R_{\mathrm{m}}+R_{\mathrm{g}}\right)
$$

where $\Delta P, R_{\mathrm{m}}$ and $R_{\mathrm{g}}$ are the pressure difference across the membrane, membrane permeate resistance and gel layer permeate resistance, respectively. The value of $R_{\mathrm{g}}$ was measured experimentally and correlated with the concentration of the gel layer, $C_{\mathrm{g}}$, and the following equations were obtained.

For ovalbumin

$$
R_{\mathrm{g}}=4.5 \times 10^{9} C_{\mathrm{g}}^{1.7}
$$

For polyvinylalcohol

$$
R_{\mathrm{g}}=2.0 \times 10^{10} C_{\mathrm{g}}^{1.7}
$$

There was an empirical law of 1.7 power regardless of solute, the characteristics and diameter of the membrane, trans membrane pressure, feed flow rate and feed concentration. Therefore, permeate flux values during ultrafiltration of ovalbumin or polyvinylalcohol can be estimated under any operation conditions using Eqs. (1), (2), and (3) or (4). The mass transfer coefficient, $k$, is determined with a correlation equation such as the Deissler correlation (Deissler, 1955), from a given feed flow rate. Although Eqs. (3) and (4) give useful relationships for estimation of permeate flux, the relationships are empirical and have not been investigated theoretically.

On the other hand, our previous work (Nabetani et al., 1990) revealed that the gel-like layer observed on the membrane surface after ultrafiltration of ovalbumin solution provides no resistance against permeation of solvents. This result is inconsistent with the conventional gel polarization model expressed with Eqs. (1), (2) and (3). In order to ensure the validity of the osmotic pressure and adsorption resistance model proposed in our previous work, it is necessary to theoretically explain the empirical law of 1.7 power based on the osmotic pressure and adsorption resistance model.

The rejection ability of the gel-like layer formed on microfiltration or ultrafiltration membranes for ovalbumin itself and other smaller solutes, such as vitamin $\mathrm{B}_{12}$ (molecular weight: 1,355), has been previously reported (Matsumoto et al., 1987; Nakao and Kimura, 1981; Nakao et al., 1982; Ohtani et al., 1985, 1988). For example, Nakao et al. (1982) treated ultrafiltration membranes with a single solute solution, consisting of vitamin $\mathrm{B}_{12}$, and a binary solute solution, consisting of both ovalbumin and vitamin $\mathrm{B}_{12}$. Although the membrane intrinsically showed no rejection ability against vitamin $B_{12}$ in an experiment with the single solute solution, the binary solute solution showed a rejection value of 80 - 90\% against vitamin $\mathrm{B}_{12}$, implying that the gel-like layer formed with ovalbumin on the membrane surface exhibited rejection ability against other solutes. Subsequently, they quantitatively analyzed the rejection ability of the gel-like layer based on the steric hindrance-pore model. Additionally, many studies were undertaken in the development of dynamically formed ultrafiltration membranes (Ohtani et al., 1985, 1987, 1988, 1989; Watanabe et al., 1986a, 1986b, 1988, 1990; Matsumoto et al., 1987; Shoji et al., 1988; Ito et al., 1990). These membranes were formed with macromolecules on a supporting medium, such as a microfiltration membrane, by utilizing the rejection ability of a gel-like layer consisting of the macromolecules.

How long does it take to reach solute adsorption equilibrium in ultrafiltration? When the membranes were soaked in an ovalbumin solution, it took a few days for the solute adsorption to reach equilibrium. However, in the filtration experiment, a constant value of permeate flux was obtained within 1-2 h, implying that adsorption had reached an equilibrium (Nabetani et al., 1990).

In order to answer these two questions, the characteristics of the gel-like layer were investigated in this study, and the adsorption rate of ovalbumin onto ultrafiltration membranes was measured under different conditions.

From the experimental results, the gel-like layer, which exhibited rejection ability for solutes and little resistance for permeate flow, was found to be a deposit layer formed by an aggregate of denatured ovalbumin. The time taken to reach adsorption equilibrium was shortened to less than $1 \mathrm{~h}$ by forced flow of the solute into the membrane pores, suggesting a notable influence of solute adsorption into membrane pores.

\section{Materials and Methods}

Apparatus and materials Two kinds of experimental apparatus, described in the author's previous study (Nabetani et al., 1990), were used in this study. One was a batch-type cell installed with a stirrer-bar, and the other was a crossflow filtration apparatus.

Three different ultrafiltration membranes, made of polyolefine (PO), polysulfone (PS), or polyacrylonitrile (PAN), and one kind of microfiltration membrane, made of cellulose triacetate (CTA), were employed, Table 1.

Ovalbumin (molecular weight: 45,000) was supplied by Syuzui Hikotarou Syouten (Tokyo, Japan). An untreated ovalbumin aqueous solution and a supernatant solution, obtained by centrifugation $(105,000 \times g, 1 \mathrm{~h})$, were used. It is reported that ovalbumin tends to be denatured at the airwater interface (Kitabatake and Doi, 1987) and adsorption of 
Table 1. Characteristics of the membranes used in this study.

\begin{tabular}{llll}
\hline Mfr. & Type & Membrane material & $\begin{array}{l}\text { MWCO or } \\
\text { pore size }\end{array}$ \\
\hline NITTO & NTU-2120 & Polyolefine (PO) & 20,000 \\
NITTO & NTU-3150 & Polysulfone (PS) & 50,000 \\
DAICEL & DUY-HH & Polyacrylonitrile (PAN) & 5,000 \\
FUJI & FM-45 & Cellulose triacetate (CTA) & $0.45 \mu \mathrm{m}$ \\
\hline
\end{tabular}

protein to the air-water interface is affected by hydrophobic interactions. Therefore, $0.1 \mathrm{M} \mathrm{NaCl}$ was used in the supernatant solution to reduce the denaturation of ovalbumin after centrifugation.

Particle size in the untreated ovalbumin solution and its supernatant solution was analyzed by laser light scattering (Sub-micron particle analyzer, type 4400, MALVERN, Worcestershire, UK). The concentrations of these solutions were measured with a total organic carbon analyzer and spectrophotometerically at $213 \mathrm{~nm}$.

Experimental conditions and procedures Two kinds of experiments were carried out. The untreated ovalbumin solution and its supernatant solution were filtrated using a crossflow filtration apparatus. The experimental conditions were as follows:

Feed concentration: 0.1 wt. $\%$

Pressure: 100, 200, 300, $400 \mathrm{kPa}$

Flow velocity: $0.45 \mathrm{~m} \mathrm{~s}^{-1}$ (corresponding to Reynolds number of 1,560$)$

\section{Feed temperature: $298 \mathrm{~K}$}

Two different kinds of ultrafiltration membranes, made of PO and PAN, and one kind of microfiltration membrane, made of CTA, were employed, Table 1. The permeate fluxes and observed rejections were measured using each filtration condition at steady state. The concentrations of feed, $C_{\mathrm{b}}$, and permeate, $C_{\mathrm{p}}$, were analyzed spectrophotometorically at 213 $\mathrm{nm}$ and the observed rejection, $R_{\mathrm{obs}}$, was calculated using Eq. (5).

$$
R_{\mathrm{obs}}=1-C_{\mathrm{p}} / C_{\mathrm{b}}
$$

In the other experiment, the decrease in pure water permeate flux with soaking time or filtration time for an untreated ovalbumin solution was measured using a batch-type cell. A flowsheet of the experiment is shown in Fig. 1. A new membrane was placed in the cell, and the intrinsic pure water permeate flux, $J_{\mathrm{w}}$, was measured. To measure the flux decline with soaking time, the membrane was removed from the cell and soaked in an ovalbumin solution. The membrane was again placed in the cell and the pure water permeate flux was measured. The procedure was repeated until a constant flux value was obtained. The flux decline with filtration time was measured as follows. After measuring $J_{\mathrm{w}}$, pure water was replaced with an ovalbumin solution and the solution
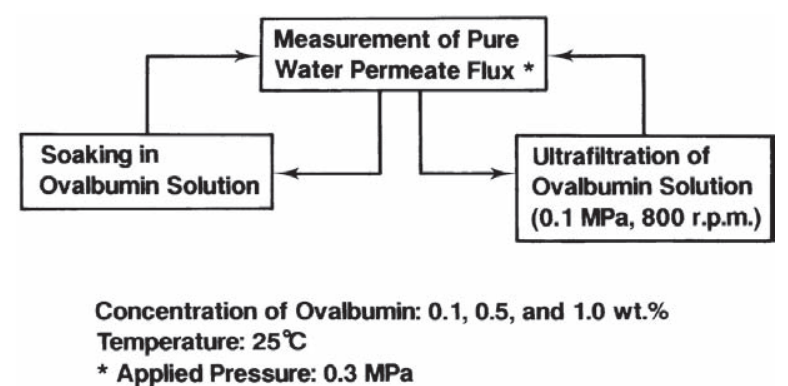

Fig. 1. Measurement of the decrease in pure water permeate flux with soaking time or filtration time for an ovalbumin solution.

was filtered under an applied pressure of $100 \mathrm{kPa}$ and stirring speed of $800 \mathrm{rpm}$ for a specified time. The solution was then replaced with pure water and the flux was measured again. This procedure was repeated until a steady state was obtained. Three different kinds of ultrafiltration membranes, made of PO, PS, and PAN, were used in this experiment (Table 1). The temperatures of the ovalbumin solution and pure water were controlled at $298 \mathrm{~K}$ and the pressure for the measurement of the pure water permeate flux was maintained at $300 \mathrm{kPa}$ with nitrogen gas. Three concentrations of ovalbumin were employed, $0.1,0.5$, and $1.0 \mathrm{wt} . \%$.

\section{Results and Discussion}

Centrifugation of ovalbumin solution Centrifugation of the ovalbumin solution gave precipitates that appeared similar to the gel-like layer observed on the membrane surface after ultrafiltration.

Analysis by laser light scattering detected particles, the diameter of which were $0.4 \mu \mathrm{m}$, in the untreated solution. These particles were not detected in the supernatant solution.

The total organic carbon contained in the supernatant solution was $96 \%$ of that in the untreated solution; although centrifugation caused little change in the absorbance at 213 $\mathrm{nm}$.

Ultrafiltration of the ovalbumin solution The fluxes, $J_{\mathrm{v}}$, and observed rejection, $R_{\mathrm{obs}}$, obtained in the ultrafiltration of untreated ovalbumin solution and its supernatant, after centrifugation, are shown in Figs. 2 and 3, respectively, where $\Delta P$ is the applied pressure. In the case of the supernatant solution, $0.1 \mathrm{M} \mathrm{NaCl}$ was used as the solvent. The lines in flux vs. pressure plots are calculated using the osmotic pressure and adsorption resistance model (Eqs. (6) and (7)) (Nabetani et al., 1990),

$$
\begin{aligned}
& J_{\mathrm{v}}=L_{\mathrm{p}}{ }^{\prime}\left\{\Delta P-\Pi\left(C_{\mathrm{m}}\right)\right\}=\left\{\Delta P-\Pi\left(C_{\mathrm{m}}\right)\right\} / R_{\mathrm{m}}{ }^{\prime} \\
& C_{\mathrm{m}}=C_{\mathrm{b}} \exp \left(J_{\mathrm{v}} / k\right)
\end{aligned}
$$

where $L_{\mathrm{p}}, C_{\mathrm{m}}$ and $\Pi$ are pure water permeability decreased by solute adsorption, solute concentration at the mem- 


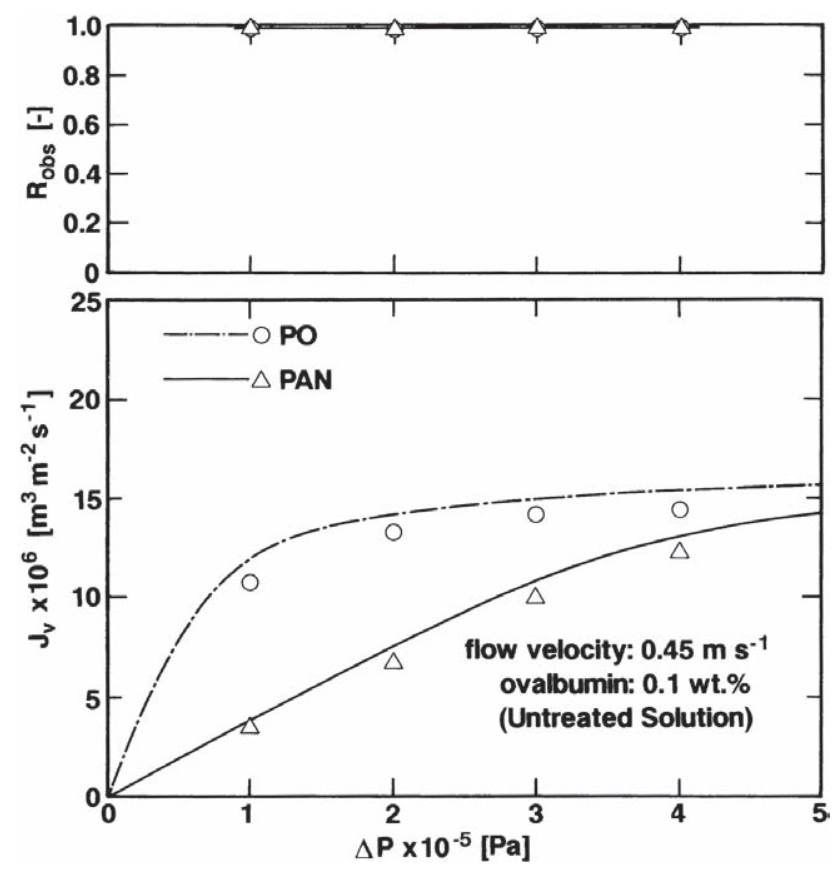

Fig. 2. Effects of applied pressure, $\Delta P$, on the permeate flux, $J_{\mathrm{v}}$, and observed rejection, $R_{\mathrm{obs}}$, during the ultrafiltration of an untreated ovalbumin solution. (Lines in $J_{\mathrm{v}}$ vs. $\Delta P$ plot were obtained using the osmotic pressure and adsorption resistance model).

brane surface and osmotic pressure, respectively, while $R_{\mathrm{m}}$ ' $\left(=1 / L_{\mathrm{p}}{ }^{\prime}\right)$ is resistance of membrane increased by solute adsorption. The experimental results for both solutions were in good agreement with calculated values. With the PAN membrane, both experimental and calculated flux values obtained in the supernatant solution were larger than those in the untreated solutions. This indicates that the addition of $\mathrm{NaCl}$ decreases solute adsorption. After ultrafiltration of the supernatant solution, a gel-like layer was not observed on the membrane surface, while a layer was observed with the untreated solution.

From these results, the gel-like layer was found to be formed with particles that could be precipitated by centrifugation, and to have little influence on permeate flux.

Microfiltration of the ovalbumin solution The microfiltration results for the two kinds of ovalbumin solution are shown in Fig. 4 , where the $J_{\mathrm{v}}$ vs. $\Delta P$ curve was calculated using the osmotic pressure and adsorption resistance model. In the untreated solution, observed rejections, $R_{\mathrm{obs}}$, of 0.9 were obtained and the formation of a self-rejection type of dynamic membrane (Ohtani et al., 1985, 1987, 1988; Watanabe et al., 1986a, 1986b, 1988, 1990; Matsumoto et al., 1987; Shoji et al., 1988; Ito et al., 1990) was observed. Experimental $J_{\mathrm{v}}$ values agreed with the calculated values, and a gel-like layer was recognized on the membrane surface at the end of the experiment. Conversely, in the supernatant solution, $R_{\mathrm{obs}}$ was zero and a gel-like layer was not observed. While $J_{v}$ de-

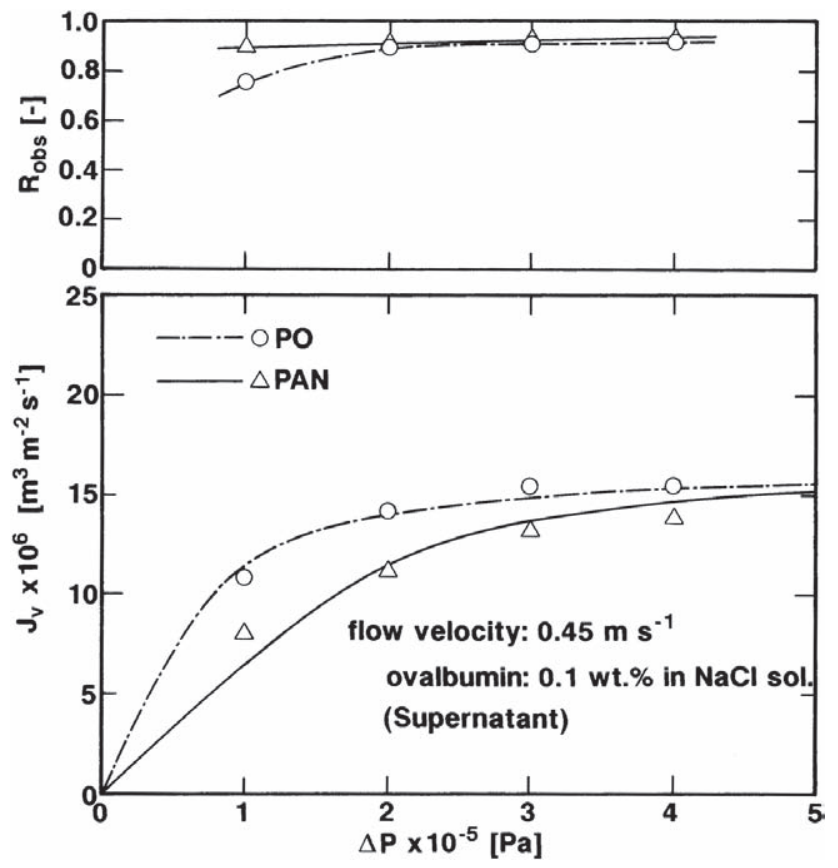

Fig. 3. Effects of applied pressure, $\Delta P$, on permeate flux, $J_{v}$, and observed rejection, $R_{\text {obs }}$, during ultrafiltration of the supernatant solution. (Lines in $J_{\mathrm{v}}$ vs. $\Delta P$ plot were obtained using the osmotic pressure and adsorption resistance model.)

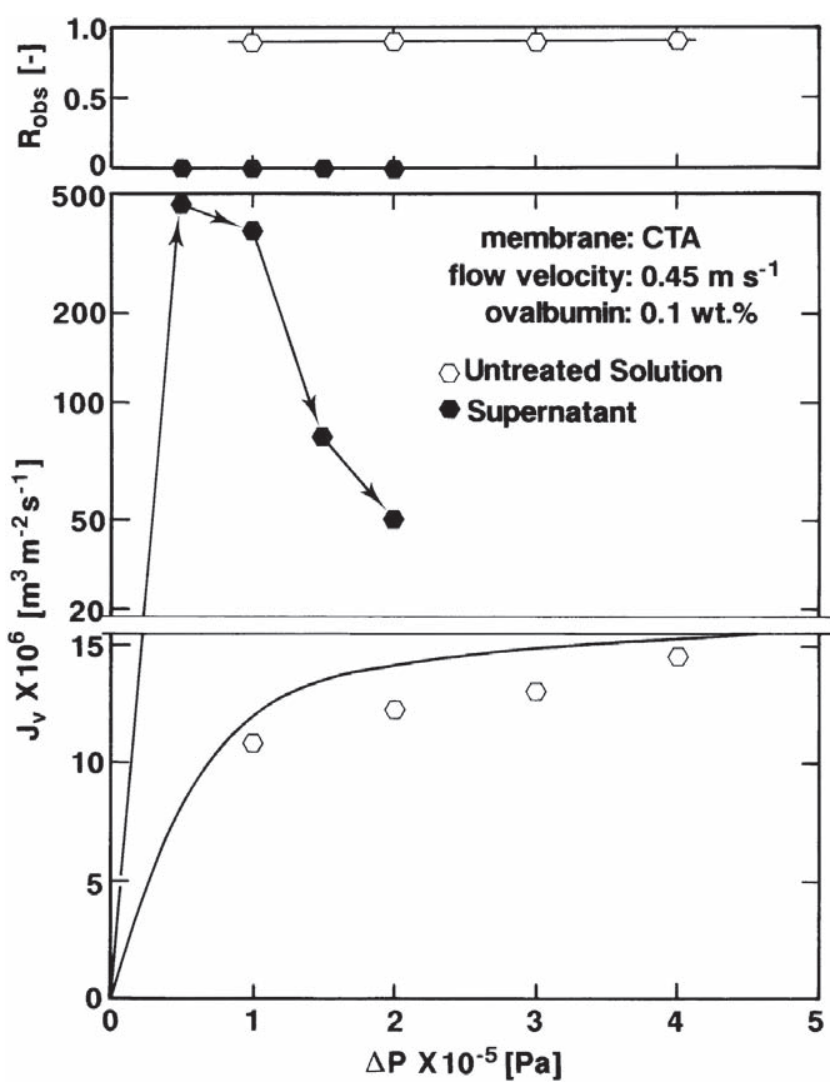

Fig. 4. Effects of applied pressure, $\Delta P$, on the permeate flux, $J_{v}$, and observed rejection, $R_{\text {obs }}$, during the microfiltration of an untreated ovalbumin solution and its supernatant solution. (A solid curve in $J_{\mathrm{v}}$ vs. $\Delta P$ plot was obtained using the osmotic pressure and adsorption resistance model.) 
creased with increasing $\Delta P$, constant $J_{\mathrm{v}}$ values were obtained at each filtration condition and were much larger than those for the untreated solution.

From these results, the reported rejection ability of the gel-like layer for ovalbumin itself and other smaller solutes (Nakao and Kimura, 1981; Nakao et al., 1982; Ohtani et al., 1985, 1988; Matsumoto et al., 1987) was found to be caused by the particles in the untreated solution.

Kitabatake and Doi (1987) reported that ovalbumin is easily denatured at the air-water interface and forms an aggregate that can be precipitated by centrifugation. The particles in the ovalbumin solution that form a gel-like layer on the membrane surface were, therefore, found to be an aggregate of denatured ovalbumin. Analysis of total organic carbon indicated that the amount of denatured ovalbumin was $4 \%$ of the total in solution.

We believe that the decrease in $J_{\mathrm{v}}$ with increasing $\Delta P$, which was observed during microfiltration of the supernatant solution, was caused by particles formed during the experiment that plug membrane pores. Changing pressure produced bubbles, which enhanced the denaturation of ovalbumin.

The supernatant of the $0.1 \mathrm{wt} . \%$ ovalbumin in water was also treated with a microfiltration membrane. Both permeate flux and observed rejection were similar to those obtained with untreated ovalbumin solution, and a slight gel-like layer was observed on the membrane surface after the experiment. These results might be caused by an aggregate that was formed at the air-water interface after centrifugation.

Koutake et al. (1987) reported that a visible gel-like layer was observed on the membrane surface after the ultrafiltration of reconstituted skim milk, but not after ultrafiltration of fresh skim milk, and the fluxes for both solutions were almost identical. Their results may be explained as follows. The drying of skim milk makes its proteins unstable. Reconstituted skim milk, therefore, contains an aggregate of denatured proteins and a gel-like layer is formed. However, the layer has little resistance to permeate flow, and flux values similar to fresh skim milk are obtained.

Relationship between permeate resistance of gel layer and concentration of the layer The results described above revealed that the gel-like layer observed on the membrane surface did not affect permeate flux. On the other hand, Nakao et al. (1979) reported that permeate resistance of gel layer, $R_{\mathrm{g}}$, was proportional to concentration of the gel layer, $C_{\mathrm{g}}$, to the power of 1.7 (Eq. (3)). In the following discussion, the empirical law of 1.7 power (Eq. (3)) is analyzed based on the osmotic pressure and adsorption resistance model.

The osmotic pressure and adsorption resistance model assumes that effective driving pressure is reduced by the osmotic pressure difference across the membrane (Eq. (6)). Nakao et al. (1979) used membranes made of cellulose acetate whose resistance was hardly affected by adsorption of proteins (Matthiasson, 1983). Therefore, the increase in membrane resistance caused by solute adsorption was assumed to be negligible $\left(R_{\mathrm{m}}{ }^{\prime} \approx R_{\mathrm{m}}\right)$. By substituting the decrease in effective driving pressure with the increase in resistance of boundary layer against solvent permeation, $R_{\mathrm{bl}}$, and disregarding the increase in membrane resistance caused by solute adsorption, Eq. (6) can be rewritten as

$$
J_{\mathrm{v}}=\Delta P /\left(R_{\mathrm{m}}+R_{\mathrm{bl}}\right)
$$

where $R_{\mathrm{b} 1}$ is expressed as

$$
R_{\mathrm{bl}}=\Pi\left(C_{\mathrm{m}}\right) R_{\mathrm{m}} /\left\{\Delta P-\Pi\left(C_{\mathrm{m}}\right)\right\}
$$

By comparing Eq. (2) with Eq. (8), the empirical law of 1.7 power observed between $C_{\mathrm{g}}$ and $R_{\mathrm{g}}$ (or $R_{\mathrm{bl}}$ ) was analyzed.

First, it was assumed that the concentration of gel layer measured by Nakao et al. (1979) represented the concentration of solute at the membrane surface, $C_{\mathrm{m}}$, and the relationship between $C_{\mathrm{m}}$ and $R_{\mathrm{bl}}$ was calculated using Eq. (10). The calculated result (dashed line in Fig. 5) differed greatly from the relationship reported by Nakao et al. (1997) (dasheddotted line in Fig. 5).

Subsequently, it was assumed that the concentration of gel layer measured by Nakao et al. represented the average concentration of solute in the concentration polarization layer. The concentration polarization equation (Eq. (7))

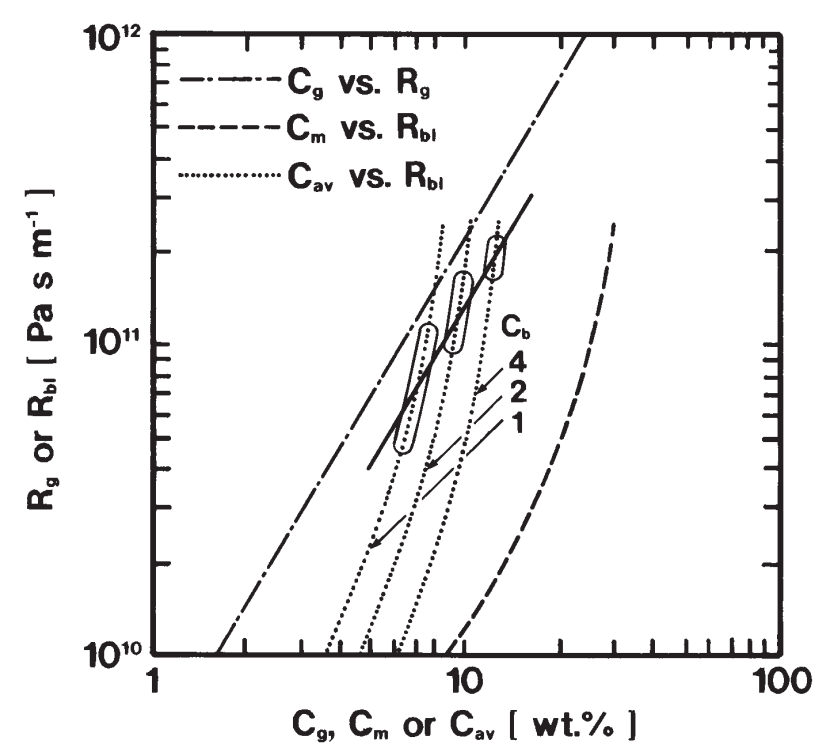

Fig. 5. The relationship between resistance of gel layer, $R_{\mathrm{g}}$, and concentration of gel layer, $C_{\mathrm{g}}$, reported by Nakao et al. (1979), and the relationships between resistance of boundary layer, $R_{\mathrm{bl}}$, and solute concentration at the membrane surface, $C_{\mathrm{m}}$, or average solute concentration in the boundary layer calculated by the osmotic pressure and solute adsorption model (Nabetani et al., 1990). 
gives the average concentration of solute in the concentration polarization layer, $C_{\mathrm{av}}$, as logarithmic mean of $C_{\mathrm{b}}$ and $C_{\mathrm{m}}$.

$$
C_{\mathrm{av}}=\left(C_{\mathrm{m}}-C_{\mathrm{b}}\right) / \ln \left(C_{\mathrm{m}} / C_{\mathrm{b}}\right)
$$

The relationships between $C_{\mathrm{av}}$ and $R_{\mathrm{bl}}$, obtained by Eqs. (10) and (11), are shown in Fig. 5 as dotted lines. None of the lines gave a slope of 1.7. Subsequently, the filtration conditions under which Nakao et al. had conducted their experiments were chosen, and the relationships between $C_{\mathrm{av}}$ and $R_{\mathrm{bl}}$ were calculated. The calculated results are shown in Fig. 5 as area surrounded by ellipses. By connecting the ellipses, a slope of 1.7 could be obtained (solid line in Fig. 5) and the concentration value approached that obtained by Nakao et al.

Nakao et al. measured solute concentration using ultraviolet absorption photometry, which could detect dissolved ovalbumin but not the aggregate of denatured ovalbumin. Therefore, the $C_{\mathrm{g}}$ values measured by Nakao et al. might be equivalent to the average concentration in concentration polarization layer that was formed on the deposit layer consisting of denatured ovalbumin. As shown in Fig. 5, $C_{\text {av }}$ (solid line) was 1.4 times higher than $C_{\mathrm{g}}$ (dashed-dotted line). This difference may be caused by dilution with the bulk solution, which covered the concentration polarization layer, that is, the thickness of the bulk solution covering the concentration polarization layer might be virtually constant in all the experiments and the layer might be diluted at the same ratio when the layer was scratched out just after the filtration experiments.

As described above, by applying the osmotic pressure and adsorption resistance model, the physical meaning of the empirical law of 1.7 power reported by Nakao et al. (1979) could be explained theoretically.

Measurement of adsorption rate Changes in the pure water permeate flux (PWF) with soaking and filtration times are shown in Fig. 6 (PAN membrane, 0.1 wt.\% ovalbumin solution). While the flux in the soaking experiment took

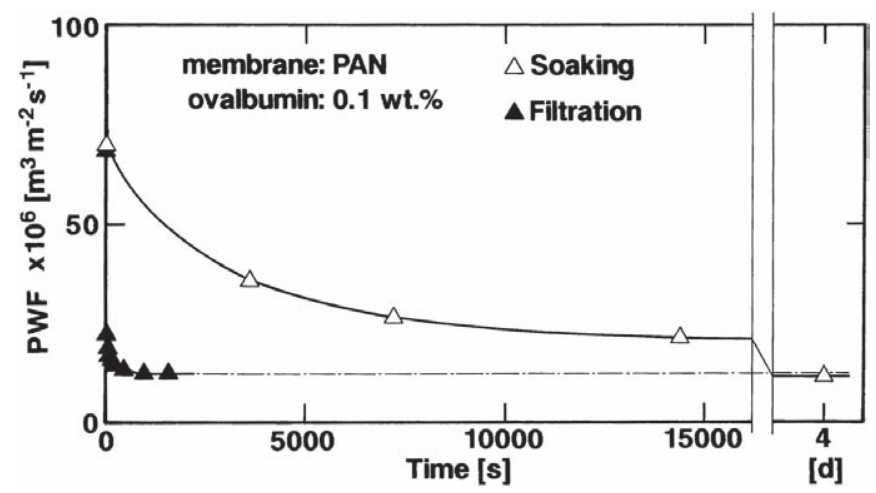

Fig. 6. Reduction in the pure water permeate flux (PWF) with soaking time and filtration time for an ovalbumin solution. about $4 \mathrm{~d}$ to reach steady state, a constant value was obtained within $2000 \mathrm{~s}$ in the filtration experiment. The constant values obtained in both experiments were almost identical. Similar results were obtained using other membranes and other concentrations of solution.

These results indicate that the forced flow of an ovalbumin solution enhances the adsorption rate, implying that the adsorption of solutes into membrane pores has a significant influence on permeate resistance. We have already reported that the resistance for pure water permeate flux caused by solute adsorption can be classified into resistance by adsorption on the surface and into pores of a membrane, that is, the resistance which can be removed by physical washing with a sponge and those that can not, respectively (Nabetani et al., 1987, 1988).

The same constant flux values obtained in the soaking experiment, $J_{\mathrm{w}, \text { ad }}$, and in the filtration experiment, $J_{\mathrm{w}, \mathrm{fil}}$, suggested that fouling phenomena, other than solute adsorption, have no effect on pure water permeability of the membrane during ultrafiltration of an ovalbumin solution.

\section{Conclusions}

An untreated ovalbumin solution and its supernatant solution, generated by centrifugation, were ultrafiltrated or microfiltrated.

A gel-like layer was always observed on the membrane surface during the ultrafiltration of an untreated solution, while such a layer was not observed with the supernatant solution. In both cases, the observed rejections approached unity, and the flux values could be predicted by the osmotic pressure and adsorption resistance model.

In the microfiltration of an untreated solution, a gel-like layer was formed. The observed rejection approached unity, and the behavior of the flux could be explained by the model. As for the supernatant solution, a gel-like layer was not formed. The observed rejection was zero, and the flux values were much larger than in the untreated solution.

It was therefore concluded that the gel-like layer, exhibiting rejection ability for ovalbumin itself and for smaller solutes, is a deposit layer formed with an aggregate of denatured ovalbumin, and can be precipitated by centrifugation. This layer has little influence on permeate flux.

While both the filtration and soaking treatments with an ovalbumin solution caused the same reductions in the pure water permeate flux for the ultrafiltration membranes, the time taken to reach adsorption equilibrium during filtration was less than $1 / 100$ of that during soaking. This implies that the adsorption of solutes into the membrane pores has a significant influence on permeate resistance. 
Acknowledgements This work was supported, in part, by a grantin-aid (Development of evaluation and management methods for supply of safe, reliable and functional food and farm produce) from the Ministry of Agriculture, Forestry and Fisheries of Japan.

\section{Nomenclature}

$C_{\text {av }}$ average concentration in concentration polarization layer [wt.\%]

$C_{\mathrm{b}} \quad$ solute concentration in bulk feed [wt.\%]

$C_{\mathrm{g}} \quad$ concentration of gel layer [wt.\%]

$C_{\mathrm{m}} \quad$ solute concentration at the membrane surface [wt.\%]

$C_{\mathrm{p}} \quad$ solute concentration in permeate [wt.\%]

$J_{\mathrm{v}} \quad$ volume flux through membrane $\left[\mathrm{m} \mathrm{s}^{-1}\right]$

$J_{\mathrm{w}} \quad$ intrinsic pure water permeate flux $\left[\mathrm{m} \mathrm{s}^{-1}\right]$

$J_{\mathrm{w}, \mathrm{ad}}$ pure water permeate flux after solute adsorption by soaking $\left[\mathrm{m} \mathrm{s}^{-1}\right]$

$J_{\mathrm{w}, \mathrm{fil}}$ pure water permeate flux after solute adsorption by filtration $\left[\mathrm{m} \mathrm{s}^{-1}\right]$

$k \quad$ mass transfer coefficient $\left[\mathrm{m} \mathrm{s}^{-1}\right]$

$L_{\mathrm{p}}$, pure water permeability decreased by solute adsorption [m $\left.\mathrm{s}^{-1} \mathrm{~Pa}^{-1}\right]$

$\Delta P$ pressure difference $[\mathrm{Pa}]$

$R_{\mathrm{bl}} \quad$ resistance of boundary layer $\left[\mathrm{Pa} \mathrm{s} \mathrm{m}^{-1}\right]$

$R_{\mathrm{g}} \quad$ resistance of gel layer $\left[\mathrm{Pa} \mathrm{s} \mathrm{\textrm {m } ^ { - 1 }}\right.$ ]

$R_{\mathrm{m}} \quad$ intrinsic membrane resistance $\left[\mathrm{Pa} \mathrm{s} \mathrm{m}{ }^{-1}\right]$

$R_{\mathrm{m}}{ }^{\prime}$ membrane resistance increased by solute adsorption [Pa s $\left.\mathrm{m}^{-1}\right]$

$R_{\text {obs }}$ observed rejection [-]

$\Pi \quad$ osmotic pressure $[\mathrm{Pa}]$

\section{References}

Baker, R. W. and Strathman, H. (1970). Ultrafiltration of macromolecular solutions with high-flux membranes. J. Applied Polym. Sci., 14, 1197-1214.

Blatt, W. F., Dravid, A., Michaels, S. and Nelson, L. (1970). Solute polarization and cake formation in membrane ultrafiltration: Causes, consequences, and control techniques. In "Membrane Science and Technology," ed. by J. E. Flinn, Plenum Press, New York, pp. 47-97.

Deissler, R. G. (1955). Analysis of turbulent heat transfer, mass transfer, and friction in Smooth tubes at high Prandtl and Schmidt Numbers. NACA Rept., 1210.

Ito, H., Nakajima, M., Nabetani, H., Ohtani, T. and Watanabe, A. (1990). Ultrafiltration of sweet-sorghum juice by polymer membranes and self-rejection type of dynamic membranes. Nippon Shokuhin Kogyo Gakkaishi, 37, 26-30 (in Japanese).

Kitabatake, N. and Doi, E. (1987). Conformational change of hen egg ovalbumin during foam formation detected by 5,5'-dithiobis (2-nitrobenzonic acid). J. Agric. Chem., 35, 953-957.

Koutake, M., Uchida, Y., Sato, T., Shimoda, K., Watanabe, A. and
Nakao, S. (1987). Filtration membrane fouling in ultrafiltration of skim milk: I. Causes and cleaning. Nippon Nogeikagaku Kaishi, 61, 677-681 (in Japanese).

Matsumoto, Y., Nakao, S. and Kimura, S. (1987). Cross-flow filtration of polymer solutions by ceramic microfiltration membranes. Kagaku Kogaku Ronbunshu, 13, 100-106 (in Japanese).

Matthiasson, E. (1983). The role of macromolecular adsorption in fouling of ultrafiltration membranes. J. Membrane Science, 16, 23-36.

Nabetani, H., Nakajima, M., Watanabe, A., Nakao, S. and Kimura, S. (1987). Effect of ovalbumin adsorption on the performance of ultrafiltration membrane. The International Congress on Membranes and Membrane Processes, Tokyo, June 8-12, pp. 451-452.

Nabetani, H., Nakajima, M., Watanabe, A., Nakao, S. and Kimura, S. (1988). Change of permeate flux and solute rejection by ovalbumin adsorption on ultrafiltration membranes. Maku (Membrane), 13, 51-57 (in Japanese).

Nabetani, H., Nakajima, M., Watanabe, A., Nakao, S. and Kimura, S. (1990). Effects of osmotic pressure and adsorption on ultrafiltration of ovalbumin. AIChE J., 36, 907-915.

Nakao, S. and Kimura, S. (1981). Effect of gel layer on rejection and fractionation of different-molecular-weight solutes by ultrafiltration. ACS Symposium Series, No. 154, American Chemical Society, Washington D. C., pp. 119-132.

Nakao, S., Nomura, T. and Kimura, S. (1979). Characteristics of macromolecular gel layer formed on ultrafiltration tubular membrane. AIChE J., 25, 615-622.

Nakao, S., Yumoto, S. and Kimura, S. (1982). Analysis of rejection characteristics of macromolecular gel layer for low molecular weight solutes in ultrafiltration. J. Chem. Eng. Jpn., 15, 463-468.

Ohtani, T., Ando, K., Tonohara, K., Ohta, H., Nawa, Y. and Watanabe, A. (1989). Clarification of Japanese Pear Juice by Inorganic Membranes. Nippon Shokuhin Kogyo Gakkaishi, 36, 448-454 (in Japanese).

Ohtani, T., Nakajima, M., Nabetani, H., Nawa, Y. and Watanabe, A. (1988). Formation and nature of ovalbumin dynamic ultrafiltration membrane. Nippon Shokuhin Kogyo Gakkaishi, 35, 807-812 (in Japanese).

Ohtani, T., Ohi, T., Horikita, H., Nakajima, M., Nabetani, H. and Watanabe, A. (1987). Recovery of $\beta$-amylase from sweet potato with self-rejection type of dynamic membrane, Nippon Shokuhin Kogyo Gakkaishi, 34, 640-646 (in Japansese).

Ohtani, T., Watanabe, A., Hoshino, C. and Kimura, S. (1985). Application of dynamic membrane to ultra-filtration. Kagaku Kogaku Ronbunshu, 11, 140-146 (in Japanese).

Porter, M. C. (1972). Concentration polarization with membrane ultrafiltration. Ind. Chem. Product Research Develop., 11, 234-248.

Shoji, T., Nakajima, M., Nabetani, H., Ohtani, T. and Watanabe, A. (1988). Effect of pore size of ceramic support on the self-rejection characteristics of the dynamic membrane formed with water 
soluble proteins in waste water. Nippon Nogeikagaku Kaishi, 62, 1055-1060 (in Japanese).

Watanabe, A., Ito, H., Nakajima, M., Nabetani, H., Ohtani, T. and Nawa, Y. (1990). Filtration of sediment from pasteurized soy sauce by self-rejection type of dynamic ultrafiltration membranes and polymer ultrafiltration membranes. Nippon Shokuhin Kogyo Gakkaishi, 37, 31-38 (in Japanese).

Watanabe, A., Komazawa, K., Nabetani, H., Nakajima, M. and Nakao, S. (1986a). Concentration of polyphenols in alkaline extracts from larch bark with self-rejection type of dynamically formed membrane. Maku (Membrane), 11, 109-114 (in Japanese).
Watanabe, A., Ohtani, T., Horikita, H., Ohya, H. and Kimura, S. (1986b). Recovery of soluble protein from fish jelly processing with self-rejection dynamic membrane. In "Food Engineering and Process Applications, vol. 2”, ed. by M. Le Manguer and P. Jelen. Elsevier Applied Science Publishers, London, pp. 225-236.

Watanabe, A., Shoji, T., Nakajima, M., Nabetani, H. and Ohtani, T. (1988). Electronmicroscopic observation of self-rejection-type of dynamic membrane formed with water soluble proteins in waste water from fish paste process. Nippon Nogeikagaku Kaishi, 62, 1061-1066 (in Japanese). 